\title{
Orientações de Enfermagem na alta hospitalar pós-procedimentos cirúrgicos
}

\section{ambulatoriais}

\author{
Nursing orientations at hospital discharge after ambulatory surgical procedures \\ Guías de Enfermería en el alta hospitalaria tras procedimientos quirúrgicos ambulatorios
}

Recebido: 03/07/2021 | Revisado: 18/07/2021 | Aceito: 21/07/2021 | Publicado: 28/07/2021

Lisiane Paula Sordi Matzenbacher

ORCID: https://orcid.org/0000-0002-1407-7717

Hospital de Clínicas de Porto Alegre, Brasil

E-mail:1sordi@hcpa.edu.br

Débora Machado Nascimento do Espírito Santo

ORCID: https://orcid.org/0000-0003-0533-0335

Hospital de Clínicas de Porto Alegre, Brasil

E-mail:dsanto@hcpa.edu.br

Carina Galvan

ORCID: https://orcid.org/0000-0003-2111-5432 Hospital de Clínicas de Porto Alegre, Brasil

E-mail:cgavan@hcpa.edu.br

Rosaura Soares Paczek

ORCID: https://orcid.org/0000-0002-4397-1814 Hospital de Clínicas de Porto Alegre, Brasil

E-mail:rspaczek@gmail.com

Ana Karina Silva da Rocha Tanaka

ORCID: https://orcid.org/0000-0003-2488-3656

Universidade Federal do Rio Grande do Sul, Brasil

E-mail:anakarinatanaka@gmail.com

\begin{abstract}
Resumo
Objetivo: descrever a prática vivenciada pelas enfermeiras de um centro cirúrgico ambulatorial, colaborando com a construção de conhecimento e o aperfeiçoamento da transição do cuidado na alta hospitalar. Método: relato de experiência, que se refere à atuação das autoras, que realizam suas atividades em um centro cirúrgico ambulatorial de um hospital público universitário de grande porte localizado no Sul do Brasil. Resultados: para que a transição do cuidado seja eficiente, além do paciente, o seu acompanhante também participa deste processo e o enfermeiro orienta sobre os cuidados específicos no pós-operatório, o uso de medicamentos e o retorno ambulatorial para reavaliação junto à equipe assistente. O paciente recebe um folder contendo todas as informações sobre os cuidados no pósoperatório e os sinais e sintomas de alerta são enfatizados tanto no folder quanto na orientação verbal realizada pelo enfermeiro. Considerações finais: a análise deste estudo permitiu identificar as principais orientações de transição do cuidado para a alta hospitalar. Ao abordar os aspectos do plano individualizado, as orientações sobre o uso de medicações e os alinhamentos de retorno para a consulta pós-procedimento, entende-se que estes são essenciais para a qualidade da assistência prestada nos serviços de saúde.
\end{abstract}

Palavras-chave: Transição para assistência do adulto; Alta do paciente; Procedimentos cirúrgicos ambulatórios.

\begin{abstract}
Objective: describe the practice experienced by nurses in an ambulatory surgical center, collaborating with the construction of knowledge and the improvement of transition of care at hospital discharge. Method: experience report, which refers to the performance of the authors, who perform their activities in an ambulatory surgical center of a large public university hospital located in the South of Brazil. Results: for the transition of care to be efficient, besides the patient, the companion also participates in this process, and the nurse provides guidance on specific postoperative care, the use of medications, and the return to the outpatient clinic for reevaluation with the assistant team. The patient receives a folder containing all the information about postoperative care, and the warning signs and symptoms are emphasized both in the folder and in the verbal orientation given by the nurse. Final considerations: the analysis of this study allowed us to identify the main care transition orientations for hospital discharge. By addressing the aspects of the individualized plan, the guidance on the use of medications, and the return alignments for the post-procedure consultation, it is understood that these are essential for the quality of care provided in health services.
\end{abstract}

Keywords: Transition to adult care; Patient discharge; Ambulatory surgical procedures. 


\begin{abstract}
Resumen
Objetivo: describir la práctica vivida por las enfermeras en un centro quirúrgico ambulatorio, colaborando con la construcción del conocimiento y mejorando la transición de la atención al alta hospitalaria. Método: relato de experiencia, que se refiere al trabajo de las autoras, quienes desarrollan sus actividades en un centro quirúrgico ambulatorio de un gran hospital universitario público ubicado en el sur de Brasil. Resultados: para que la transición de la atención sea eficiente, además del paciente, el cuidador también participa en este proceso y el enfermero brinda orientación sobre cuidados postoperatorios específicos, el uso de medicación y el retorno ambulatorio para revaloración con el equipo asistente. El paciente recibe una carpeta que contiene todas las informaciones sobre los cuidados postoperatorios y las señales de advertencia y los síntomas se enfatizan tanto en la carpeta como en la guía verbal proporcionada por el enfermero. Consideraciones finales: el análisis de este estudio permitió identificar las principales pautas para la transición de la atención al alta hospitalaria. Al abordar los aspectos del plan individualizado, las pautas sobre el uso de medicamentos y las pautas de devolución para la consulta posprocedimiento, se entiende que estos son fundamentales para la calidad de la atención brindada en los servicios de salud.
\end{abstract}

Palabras clave: Transición a la atención de adultos; Alta del paciente; Procedimientos quirúrgicos ambulatorios.

\title{
1. Introdução
}

A cirurgia ambulatorial é aquela em que o paciente é submetido a um procedimento cirúrgico, podendo ser utilizadas diversas técnicas anestésicas como: anestesia geral, regional, local ou sedação. Os procedimentos normalmente são pouco invasivos e de rápida duração, necessitando de cuidados pós-operatórios imediatos e, geralmente, a alta hospitalar ocorre no mesmo dia ou, se houver indicação da equipe cirúrgica e/ou anestésica, poderá ser necessário pernoitar na sala de recuperação pós-anestésica (Silva et al., 2018).

Os procedimentos cirúrgicos ambulatoriais possuem vantagens como: a diminuição do risco de infecções hospitalares; a redução da ansiedade pré-operatória do paciente e de seus familiares; o retorno mais rápido para o ambiente domiciliar, a vida social e as atividades laborais, contribuindo, também, na redução de custos para a instituição de saúde (Carneiro et al., 2021).

Dentro das instituições hospitalares, o médico é o responsável legal pela alta dos pacientes, porém, o enfermeiro tem papel essencial nesse planejamento, realizando orientações dos cuidados de Enfermagem de forma sistematizada e integral. Assim, no momento em que a alta é definida pela equipe médica, a Enfermagem será responsável pelas condutas de educação (Modas et al.,2019).

Neste contexto, a fragilidade das instruções no momento da alta afeta a segurança do paciente. O planejamento deve levar em consideração as dificuldades de entendimento às quais o paciente está sujeito por não conhecer ou vivenciar aquela prática. As orientações são cruciais para garantir uma transição segura e eficaz (Fontana et al.,2017; Silva, et al.,2015).

A transição é definida como um grupo de ações planejadas para garantir a continuidade do cuidado quando os pacientes são transferidos de uma unidade para outra ou até mesmo para o seu domicílio. Estratégias bem estabelecidas têm impacto na redução de internações hospitalares e na diminuição das reinternações causadas por complicações (Okafo et al.,2017; Menezes et al.,2019).

Este relato de experiência tem como objetivo descrever a prática vivenciada pelas enfermeiras de um centro cirúrgico ambulatorial, colaborando com a construção de conhecimento e o aperfeiçoamento da transição do cuidado na alta hospitalar.

\section{Metodologia}

Trata-se de um estudo descritivo, realizado em junho de 2021, do tipo relato de experiência, que se refere à atuação das autoras deste estudo, que realizam suas atividades laborais em um centro cirúrgico ambulatorial de um hospital público universitário de grande porte localizado no Sul do Brasil. O centro cirúrgico ambulatorial conta com oito salas de cirurgias onde são realizados procedimentos de diversas especialidades de pequeno e médio porte, cinco salas de procedimentos 
endoscópicos, quinze leitos de recuperação pós-anestésica adultos, cinco leitos de recuperação pediátricos e oito leitos para a recuperação pós-sedação de procedimentos endoscópicos.

Este relato será reproduzido e descrito, em detalhes, por meio da realização da prática assistencial sistematizada de modo a esclarecer e a demonstrar o trabalho diário do enfermeiro de uma sala de recuperação pós-anestésica, abrangendo os processos, os protocolos e as produções científicas, implementando seus conhecimentos e aplicando a prática baseada em evidências, desafiando a desenvolver seus conhecimentos técnicos-científicos por meio da pesquisa, das metodologias e das relações multidisciplinares (Tannure et al., 2010; Minayo,2012).

\section{Relato da Experiência}

A recuperação pós-procedimento compreende uma etapa muito importante iniciada na sala de recuperação e estendida para o domicílio do paciente. A primeira avaliação para a alta após a cirurgia ambulatorial é realizada pelo enfermeiro, que utiliza critérios pré-definidos e sistematizados por meio de protocolos assistenciais. Estes são essenciais para padronizar as atividades e garantir a qualidade nas condutas, seguindo um padrão por todos os enfermeiros que as executam dentro da sala de recuperação.

O paciente é avaliado constantemente, utilizando critérios que têm o objetivo de sistematizar a assistência de Enfermagem, observando as condições fisiológicas, assim como os efeitos residuais dos fármacos administrados durante a cirurgia. Avaliam-se também a frequência respiratória e a expansibilidade torácica, a oxigenação por meio do oxímetro de pulso, atentando-se aos níveis de oxigenação com ou sem suplementação de oxigênio. A circulação, a frequência cardíaca, os níveis pressóricos, a coloração da pele, a atividade e a resposta a estímulos com movimentações sob comando e o nível de consciência orientado quanto ao tempo e espaço também são avaliados e devem estar completamente estabelecidos para que o paciente possa receber a alta hospitalar.

É realizada uma segunda avaliação em que se observam a readaptação ao ambiente, como sentar, levantar e caminhar, a presença de diurese espontânea, conforme a abordagem cirúrgica, a ausência de náuseas e vômitos, a dor controlada e a aceitação de dieta via oral. Com o paciente atendendo a todos os critérios de alta descritos, ele é liberado pela equipe médica e o enfermeiro realiza as orientações de alta hospitalar.

No primeiro dia, é recomendado que o paciente faça repouso. Por isso, é importante a participação do acompanhante/família no cuidado para o seu conforto. No decorrer dos dias, ele poderá retomar suas atividades gradualmente. Ele ainda deve evitar fazer esforços e carregar peso até a liberação para atividades físicas pela equipe médica, reforçando a importância da realização gradual das atividades diárias de forma independente. Salientam-se aquelas atividades que exigem maior atenção, como, por exemplo, mexer em fogão, operar máquinas ou dirigir, pois sua atenção estará diminuída devido aos medicamentos anestésicos que recebeu e ainda interferem nas reações imediatas. Caso apresente alguma dúvida, poderá entrar em contato com a enfermeira do hospital para novos esclarecimentos.

Para que a transição do cuidado seja eficiente, além do paciente, o seu acompanhante também participa deste processo e o enfermeiro orienta sobre os cuidados a serem tomados com a prescrição de medicamentos, esclarecendo eventuais dúvidas remanescentes como sobre a frequência de uso. Reforça que seus medicamentos de uso regular, ou seja, os quais já fazia uso antes da cirurgia, devem ser retomados nos horários já preconizados. Caso tenha deixado de tomar alguma medicação em virtude do preparo e da realização da cirurgia, deverá retornar o uso sem necessidade de compensar, tomando a dose não ingerida, fazendo uso apenas da medicação do horário.

Esclarece-se também sobre a dor pós-operatória, que é normal sentir um desconforto e dor nos primeiros dias após a cirurgia, devido ao procedimento. Dores muito fortes e que não aliviam com os medicamentos prescritos são sinais de alerta. $\mathrm{O}$ 
paciente é orientado a entrar em contato com a equipe que realizou a cirurgia ou procurar o serviço de emergência do hospital para ser reavaliado.

Se for necessário ir para o domicílio com algum dreno, cateter ou sonda, o paciente é orientado quanto aos cuidados na manipulação, esvaziamento, cuidados com os clamps e higienização do pertuito e do reservatório. Quanto ao curativo cirúrgico, seguem-se as orientações conforme a recomendação médica e, caso haja pontos para serem retirados, os mesmos devem ser retirados no retorno médico. Se o retorno médico ocorrer após 30 dias da realização da cirurgia, orienta-se o paciente a procurar uma Unidade Básica de Saúde para que seja avaliado e os pontos sejam retirados após dez a 12 dias do procedimento.

O paciente recebe um folder contendo todas as informações sobre os cuidados no pós-operatório e os sinais e sintomas de alerta são enfatizados tanto no folder quanto na orientação verbal realizada pelo enfermeiro. O paciente deverá retornar ao serviço de saúde caso apresente alguma das intercorrências descritas no folder como febre, dor muito forte que não melhore com o uso de medicamentos, náuseas e vômitos, sangramento ou sinais de infecção. No momento da alta, o paciente é orientado a agendar a sua consulta de retorno para ser reavaliado pela equipe assistente.

\section{Discussão}

A alta hospitalar é caracterizada pela transição do cuidado entre profissional e o manejo domiciliar e pode ser assinalada como um período de vulnerabilidade para o paciente. Um instante em que as orientações são realizadas, as dúvidas, sanadas e o plano terapêutico é traçado, tido como uma fase crítica (Silva et al., 2018; SOBECC,2017).

A educação do paciente deve ser realizada de forma clara, com linguagem objetiva, por escrito, contendo medicações, orientações de cuidados, consulta de retorno e explicações sobre sinais e sintomas de complicações, que são instruções que fortalecem o aprendizado. Seguir, de forma correta, as orientações depende do processo de educação para a alta hospitalar (Camargo et al.,2016; Fontana et al., 2017).

O planejamento de cuidados deve iniciar-se ainda dentro da unidade hospitalar e as atividades de educação e promoção do autocuidado são primordiais para garantir que as orientações sejam transmitidas de forma clara entre o profissional de saúde e o paciente. Quanto às instruções de autogerenciamento do cuidado, identificam-se ações educacionais relacionadas ao uso de medicamentos, à dieta adequada e à necessidade de plano de cuidado individual (Costa et al.,2015; Suter et al.,2017).

A administração correta de medicamentos deve ser um dos focos primários realizados pela equipe no momento da alta. As drogas utilizadas devem ser descritas e o uso do método do feedback para verificar a compreensão a respeito do uso seguro é essencial, contribuindo para avaliar a compreensão do paciente diante das informações recebidas e reduzir a probabilidade de erros (Lima et al.,2018; Figueiredo et al.,2016; Garcia et al.,2017).

Os apontamentos descritos aqui sugerem que os pacientes que recebem informações sobre a transição do cuidado, de forma clara e objetiva, têm uma probabilidade significativamente menor de ser readmitidos no hospital devido a complicações. A entrega de um guia com orientações é um facilitador para que o paciente e a família possam assumir a responsabilidade pelo gerenciamento e a educação do autocuidado (Hung et al.,2015; Occelli et al.,2016).

O acompanhamento ambulatorial desses pacientes é crucial para a continuidade do cuidado. Essas ações podem identificar complicações precocemente, prevenindo reinternações e sendo essenciais para um planejamento eficaz. Um processo de transição eficiente requer um olhar individualizado do profissional de saúde, com comunicação eficaz entre a unidade hospitalar e o paciente, colaborando para minimizar eventos adversos (Hirschman et al.,2015; Weber et al.,2017). 
As limitações deste estudo estão relacionadas a ter sido realizado em uma instituição de ensino e, por este motivo, os profissionais envolvidos possuem suas funções claramente elucidadas, contribuindo para a definição de que as demandas de cuidados são atribuições do enfermeiro.

\section{Considerações Finais}

A análise deste estudo permitiu identificar as principais orientações de transição do cuidado para a alta hospitalar. Ao abordar os aspectos do plano individualizado, as orientações sobre o uso de medicações e os alinhamentos de retorno para a consulta pós-procedimento, entende-se que estes são essenciais para a qualidade da assistência prestada nos serviços de saúde.

Portanto, este estudo fornece informações importantes, aprimorando as práticas assistenciais e a sistematização das atividades do enfermeiro, que assume a função de coordenador da alta, oferecendo suporte para que pacientes e familiares possam realizar seus cuidados com autonomia e segurança. Sugere-se futuras pesquisas nesta temática para endossar os conhecimentos nesta área.

\section{Referências}

Camargo, P., André, L \& Lamari, N. (2016). Orientações em saúde no processo de alta hospitalar em usuários reinternados do Sistema Único de Saúde. Arquivos De Ciências Da Saúde, 23(3), 38-43. https://doi.org/10.17696/2318-3691.23.3.2016.335.

Carneiro, J. M. de Jesus, L. O. Silva, C. S., Santiago, A. S., Santos, A. A. L., \& Marques, P. F. (2021). Nursing discharge plan in hospitals: an experience report / Plano de alta de enfermagem no contexto hospitalar: um relato de experiência. Revista De Pesquisa Cuidado é Fundamental Online, 12, 1045-1049. https://doi.org/10.9789/2175-5361.rpcfo.v12.7495.

Costa, T. M. N. \& Sampaio, C. E. P.(2015). As orientações de enfermagem e sua influência nos níveis de ansiedade dos pacientes cirúrgicos hospitalares. Rev Enferm UERJ, Rio de Janeiro; 23(2): 260-265. https://doi.org/10.12957/reuerj.2015.16534.

Figueiredo, T. P., Carmo, Jr. N. M., Groia, R. C. S.,Pereira, R. C. C.,Silveira, R. R., Malta, J. S. \& Costa, J. M. (2016).Análise das intervenções realizadas na alta hospitalar de idosos acompanhados em serviço de orientação e referenciamento farmacoterapêutico. Rev APS.19(3):376-83.

Fontana, G., Chesani, F. H.\& Nalin, F.(2017). Percepções dos profissionais da saúde sobre o processo de alta hospitalar. Revista da UNIFEBE, 1 (21),137-156.

Garcia, J. B. S., Bonilla P., Kraychetec, D. C., Flores, F. C., Valtolina, E. D. P. \& Guerrero, C. (2017). Aprimorar o controle da dor no pós-operatório na América Latina. RevBras Anestesiol. ,67(4):395-403.https://doi.org/10.1016/j.bjane.2016.04.003.

Hirschman, K. B., Shaid, E., McCauley, K., Pauly, M. V. \& Naylor, M. D.(2015). Continuity of care: the Transitional Care Model. Online journal of issues in nursing, 20(3), 1 .

Hung, D. \& Leidig, R. C. (2015). Implementing a Transitional Care Program to Reduce Hospital Readmissions Among Older Adults. J Nurs Care Qual. ,30(2):121-9.

Lima, M. A. D. S., Magalhãe, A. M. M., Oelke, N. D., Marques, G. Q., Lorenzini, E., Weber, L. A. \& Fan,I. (2018).Care transition strategies in Latin American countries: an integrative review. Revista Gaúcha de Enfermagem, 39, e20180119. https://doi.org/10.1590/1983-1447.2018.20180119.

Menezes, T. M. O.,Oliveira, A. L. B.,Santos,L. B.,Freitas,R. A.,Pedreira,L. C. \& Veras,S. M. C. B. (2019).Hospital transition care for the elderly: an integrative review. Revista Brasileira de Enfermagem ,72(2), 294-301. doi.org/10.1590/0034-7167-2018-0286.

Minayo, M. C. S. (2012). Análise qualitativa: teoria, passos e fidedignidade. Ciência \& Saúde Coletiva. 17(3) , 621-626. https://doi.org/10.1590/S141381232012000300007

Modas, D. A. S.,Nunes, E. M. G. T. \& Charepe, Z. B. (2019). Causas de atraso na alta hospitalar no cliente adulto: scoping review. Revisão Sistemática. Rev. Gaúcha Enferm. 40. https://doi.org/10.1590/1983-1447.2019.20180130.

Occelli, P., Touzet, S., Rabilloud, M., Ganne, C., Poupon Bourdy, S., Galamand, B., Debray, M., Dartiguepeyrou, A., Chuzeville, M., Comte, B., Turkie, B., Tardy, M., Luiggi, J. S., Jacquet-Francillon, T., Gilbert, T., \& Bonnefoy, M. (2016). Impact of a transition nurse program on the prevention of thirty-day hospital readmissions of elderly patients discharged from short-stay units: study protocol of the PROUST stepped-wedge cluster randomised trial. BMC geriatrics, 16, 57. https://doi.org/10.1186/s12877-016-0233-2.

Okafor, N., Mazzillo, J., Miller, S., Chambers, K.A., Yusuf, S., Garza-Miranda, V. \& Chathampally, Y. (2017).Improved accuracy and quality of information during emergency department care transitions. West J Emerg Med., 18(3):459-65. https://doi.org/10.5811/westjem.2016.12.30858.

Silva, R., Ribeiro, M. A., \& Azevêdo, C. D. (2018). Concepções sobre o Processo de Alta Hospitalar: Uma Revisão Crítica. Tempus Actas de Saúde Coletiva, $12,135-146$

Silva, F., Junior, N., Oliveira, D., Nicoletti, D., \& Comin, E. (2015). Análise de eventos adversos em um Centro Cirúrgico ambulatorial. Revista SOBECC, 20(4), 202-209. https://revista.sobecc.org.br/sobecc/article/view/91 
Research, Society and Development, v. 10, n. 9, e37210917834, 2021

(CC BY 4.0) | ISSN 2525-3409 | DOI: http://dx.doi.org/10.33448/rsd-v10i9.17834

SOBECC(2017). Diretrizes de prática em enfermagem cirúrgica e processamento de produtos para a saúde. Associação Brasileira de Enfermagem de Centro Cirúrgico, Recuperação Anestésica e Centro de Material e Esterilização. (7a ed.), Manole

Suter, E., Oelke, N. D., Lima, M. A. D. S., Stiphout, M., Janke, R., Witt, R. R., Van Vliet-Brown, C., Schill, K., Rostami, M., Hepp, S., Birney, A., AlRoubaiai, F. \& Marques, G. M. (2017). Indicators and Measurement Tools for Health Systems Integration: A Knowledge Synthesis. International Journal of Integrated Care, 17(6), 4. DOI: http://doi.org/10.5334/ijic.3931.

Tannure, M. C.\& Pinheiro, A. M.(2010). Sistematização da Assistência de Enfermagem: guia prático. (2a ed.), Guanabara Koogan ed.

Weber, L. A. F., Lima, M. A. D. S. A, Costa, A. M. \& Marques, G. Q. (2017). Care transition from hospital to home: integrative review. Cogitare Enferm.,(22)3:47615.http://dx.doi.org/10.5380/ce.v22i3.47615. 